\title{
SHIFT BASIC SEQUENCES IN THE WIENER DISC ALGEBRA
}

\author{
J. R. HOLUB
}

\begin{abstract}
Let $W(D)$ denote the set of functions $f(z)=\sum_{n=0}^{\infty} a_{n} z^{n}$ for which $\sum_{n=0}^{\infty}\left|a_{n}\right|<+\infty$. It is shown that for any positive integer $k$ the $k$-shifted sequence $\left\{z^{k n} \cdot f(z)\right\}_{n=0}^{\infty}$ is a basic sequence in $W(D)$ equivalent to the basis $\left\{z^{n}\right\}_{n=0}^{\infty}$ if and only if $f(z)$ has no set of $k$ symmetrically distributed zeros on the circle $|z|=1$.
\end{abstract}

1. Introduction. A sequence $\left\{x_{n}\right\}_{n=0}^{\infty}$ in a Banach space $X$ is said to be a basis for $X$ if every $x \in X$ can be written uniquely as a convergent series $x=\sum_{n=0}^{\infty} a_{n} x_{n}$. The sequence $\left\{x_{n}\right\}_{n=0}^{\infty}$ is called a basic sequence [4, p. 27] if it forms a basis for its closed linear span in $X$. If $\left\{x_{n}\right\}_{n=0}^{\infty}$ is a basis for $X$ and $\left\{y_{n}\right\}_{n=0}^{\infty}$ a basis for $Y$, we say $\left\{x_{n}\right\}_{n=0}^{\infty}$ and $\left\{y_{n}\right\}_{n=0}^{\infty}$ are equivalent if the operator $T: X \rightarrow Y$ defined by $T x_{n}=y_{n}$ is a linear homeomorphism, i.e. if $\sum_{n=0}^{\infty} a_{n} x_{n}$ converges in $X$ if and only if $\sum_{n=0}^{\infty} a_{n} y_{n}$ converges in $Y[4$, p. 70].

Let $W(D)$ denote the Wiener disc algebra of all analytic functions $f(z)=\sum_{n=0}^{\infty} a_{n} z^{n}$ for which $\sum_{n=0}^{\infty}\left|a_{n}\right|<+\infty$. Defining the norm on $W(D)$ by $\|f\|=\sum_{n=0}^{\infty}\left|a_{n}\right|$ and multiplication as that of analytic functions, $W(D)$ is a Banach algebra with identity 1 which is isometrically isomorphic to the (complex) sequence space $l^{1}$ under the mapping $\sum_{n=0}^{\infty} a_{n} z^{n} \leftrightarrow\left\{a_{n}\right\}_{n=0}^{\infty}$. It follows that the sequence $\left\{z^{n}\right\}_{n=0}^{\infty}$ is a basis for $W(D)$ which is equivalent to the unit vector basis $\left\{e_{n}\right\}_{n=0}^{\infty}$ for $l^{1}$ and which is referred to as the standard basis for $W(D)$.

Now this basis $\left\{z^{n}\right\}_{n=0}^{\infty}$ is simply the set of successive shifts $\left\{1, z \cdot 1, \ldots, z^{n} \cdot 1, \ldots\right\}$ of the function $1 \in W(D)$. Thus a very natural and interesting problem which arises is that of determining the basis behavior of more general shift sequences in $W(D)$, and particularly that of distinguishing those equivalent to the standard basis $\left\{z^{n}\right\}_{n=0}^{\infty}$. In its greatest generality this latter problem can be stated as follows:

Given a function $f(z)$ in $W(D)$ and a positive integer $k$, when is the " $k$-shifted" sequence $\left\{z^{k n} \cdot f(z)\right\}_{n=0}^{\infty}$ a basic sequence in $W(D)$ equivalent to the standard basis $\left\{z^{n}\right\}_{n=0}^{\infty} ?$

The purpose of this paper is to provide a complete solution to the problem. In $\S 2$ it is shown that the sequence $\left\{z^{k n} \cdot f(z)\right\}_{n=0}^{\infty}$ is basic in $W(D)$ and equivalent to $\left\{z^{n}\right\}_{n=0}^{\infty}$ if and only if $f(z)$ has no set of $k$ zeros on the circle $|z|=1$ which are "symmetrically distributed", i.e. if $\sum_{i=1}^{k}\left|f\left(\omega^{i} z\right)\right| \neq 0$ for all $z$, where $\omega$ is a primitive $k$ th root of 1 . The solution to the general problem of characterizing those functions $f(z)$ for which $\left\{z^{k n} \cdot f(z)\right\}_{n=0}^{\infty}$ is some basic sequence in $W(D)$ (not necessarily

Received by the editors February 16, 1982 and, in revised form, November 12, 1982.

1980 Mathematics Subject Classification. Primary 46B15, 46E15, $47 \mathrm{~B} 37$.

Key words and phrases. Wiener algebra, basis, basic sequence, shift operator.

(C) 1983 American Mathematical Society 0002-9939/82/0000-1186/\$02.50 
equivalent to $\left.\left\{z^{n}\right\}_{n=0}^{\infty}\right)$ is considerably more difficult even when $k=1$, and is known at this time only in special cases, e.g., when $f(z)$ is a polynomial. We briefly discuss the problems and possibilities inherent in this general case in $\S 3$.

Finally, we note that problems of this type concerning the description of 1-shifted sequences which form bases for function spaces other than $W(D)$ have been extensively studied (see, e.g., [1] and its bibliography). In the space $W(D)$ itself it was shown earlier [2] that all shift bases for $W(D)$ are equivalent to $\left\{z^{n}\right\}_{n=0}^{\infty}$. Hence the present paper forms the natural completion to this aspect of the study of shift sequences in $W(D)$.

2. Main results. In this section we characterize those functions $f(z)$ for which a shift sequence of the form $\left\{z^{k n} \cdot f(z)\right\}_{n=0}^{\infty}$ in $W(D)$ is a basic sequence equivalent to $\left\{z^{n}\right\}_{n=0}^{\infty}$. In particular we give such a characterization in terms of the location of zeros of $f(z)$ on the unit circle $|z|=1$, thereby linking the function-theoretic properties of $f(z)$ to the basic structure of $W(D)$.

THEOREM. Let $f(z)$ be a nonzero function in $W(D)$ and $k$ a positive integer. Then the sequence $\left\{z^{k n} \cdot f(z)\right\}_{n=0}^{\infty}$ is a basic sequence in $W(D)$ equivalent to $\left\{z^{n}\right\}_{n=0}^{\infty}$ if and only if $\sum_{i=1}^{k}\left|f\left(\omega^{i} z\right)\right| \neq 0$ for all $|z|=1$, where $\omega$ is a primitive kth root of unity.

I.e. $\left\{z^{k n} \cdot f(z)\right\}_{n=0}^{\infty}$ is equivalent to $\left\{z^{n}\right\}_{n=0}^{\infty}$ if and only if $f(z)$ does not have $k$ zeros which are "symmetrically distributed" around the circle $|z|=1$.

Proof. In the interest of simplicity of notation we prove the theorem for the case $k=2$, then indicate the straightforward adaptation of the argument to all other values of $k$. Thus we will characterize the equivalence of a sequence of the form $\left\{z^{2 n} \cdot f(z)\right\}_{n=0}^{\infty}$ to $\left\{z^{n}\right\}_{n=0}^{\infty}$.

Since $f(z) \neq 0$ we may suppose $f(z)$ has the form $f(z)=1+a_{1} z+a_{2} z^{2}+\cdots$. If $\left\{z^{n}\right\}_{n=0}^{\infty}$ denotes the usual basis for $W(D)$ then the linear operator $T: W(D) \rightarrow$ $W(D)$ defined by $T\left(z^{n}\right)=z^{2 n} \cdot f(z), n=0,1,2, \ldots, \quad$ is continuous since $\sup _{n}\left\|T\left(z^{n}\right)\right\|=\|f(z)\|<+\infty$. It follows that the sequence $\left\{z^{2 n} \cdot f(z)\right\}_{n=0}^{\infty}$ is a basic sequence in $W(D)$ equivalent to $\left\{z^{n}\right\}_{n=0}^{\infty}$ if and only if there is an $\varepsilon>0$ for which $\|T(g(z))\| \geqslant \varepsilon$ for all $g(z)$ in $W(D)$ with $\|g(z)\|=1$.

If we define $f_{1}(z)=\sum_{n=0}^{\infty} a_{2 n} z^{n}$ and $f_{2}(z)=\sum_{n=0}^{\infty} a_{2 n+1} z^{n}$, then clearly for any $g(z)$ in $W(D)$ we have

$$
T(g(z))=g\left(z^{2}\right) \cdot f(z)=g\left(z^{2}\right) f_{1}\left(z^{2}\right)+z \cdot g\left(z^{2}\right) f_{2}\left(z^{2}\right) .
$$

It follows that

$$
\|T g(z)\|=\left\|g\left(z^{2}\right) \cdot f_{1}\left(z^{2}\right)\right\|+\left\|z g\left(z^{2}\right) f_{2}\left(z^{2}\right)\right\|=\left\|g(z) \cdot f_{1}(z)\right\|+\left\|g(z) \cdot f_{2}(z)\right\|,
$$

and by our comments above we have: The sequence $\left\{z^{2 n} \cdot f(z)\right\}_{n=0}^{\infty}$ is a basic sequence in $W(D)$ equivalent to $\left\{z^{n}\right\}_{n=0}^{\infty}$ if and only if there is an $\varepsilon>0$ such that $\left\|g(z) \cdot f_{1}(z)\right\|$ $+\left\|g(z) \cdot f_{2}(z)\right\| \geqslant \varepsilon$ for all $g(z)$ in $W(D)$ with $\|g(z)\|=1$.

Thus we need only show that the assumptions of the theorem imply this last holds.

Suppose, then, that the condition of the theorem is satisfied for $k=2$, i.e. $|f(z)|+|f(-z)| \neq 0$ for every $|z|=1$. It follows that $\left|f_{1}(z)\right|+\left|f_{2}(z)\right| \neq 0$ for every 
$|z|=1$, since if $f_{1}(z)=f_{2}(z)=0$, let $w=z^{1 / 2}$ so that $|w|=1$ and

$$
\begin{aligned}
f(w) & =1+a_{1} w+a_{2} w^{2}+\cdots=\left(1+a_{2} z+a_{4} z^{2}+\cdots\right)+w\left(a_{1}+a_{3} z+\cdots\right) \\
& =f_{1}(z)+w f_{2}(z)=0,
\end{aligned}
$$

while

$$
\begin{aligned}
f(-w) & =1-a_{1} w+a_{1} w^{2}-\cdots=\left(1+a_{1} z+a_{2} z^{2}+\cdots\right)-w\left(a_{1}+a_{3} z+\cdots\right) \\
& =f_{1}(z)-w f_{2}(z)=0,
\end{aligned}
$$

a contradiction to our assumption.

Now let $A$ denote the Banach algebra of all functions $f(\theta)$ on $[0,2 \pi]$ whose Fourier series is of the form $f(\theta)=\sum_{n=-\infty}^{\infty} a_{n} e^{-i n \theta}$, where $\sum_{-\infty}^{\infty}\left|a_{n}\right|<+\infty$, and for which $\|f(\theta)\|=\sum_{-\infty}^{\infty}\left|a_{n}\right|$ (see, e.g., [3, p. 195]). Clearly we may identify $W(D)$ with the subspace of $A$ consisting of those functions $f(\theta)=\sum_{-\infty}^{\infty} a_{n} e^{-i n \theta}$ for which $a_{n}=0$ for $n<0$.

Regarding $f_{1}$ and $f_{2}$ as functions in $A$, by the above we have that $f_{1}$ and $f_{2}$ have no common zero on [0,2 $\pi$ ]. Since the proper maximal ideals in $A$ are sets of the form $\left\{h \in A \mid h\left(t_{0}\right)=0\right\}$ for some $t_{0} \in[0,2 \pi]\left[3\right.$, p. 195], it follows that $f_{1}$ and $f_{2}$ are not both contained in any proper maximal ideal of $A$ and, hence, that the ideal in $A$ generated by $f_{1}$ and $f_{2}$ must be $A$ itself. In particular, there exist functions $p$ and $q$ in $A$ for which $p \cdot f_{1}+q \cdot f_{2}=1$.

Now suppose it is not true that there exists an $\varepsilon>0$ such that $\|g(z)\|=1$ implies $\left\|f_{1}(z) \cdot g(z)\right\|+\left\|f_{2}(z) \cdot g(z)\right\| \geqslant \varepsilon$. Then there exists a sequence $\left\{g_{n}(z)\right\}$ in $W(D)$ for which $\left\|g_{n}(z)\right\|=1$ for all $n$, but $\left\|g_{n}(z) \cdot f_{1}(z)\right\| \rightarrow 0$ and $\left\|g_{n}(z) \cdot f_{2}(z)\right\| \rightarrow 0$. Regarding $\left\{g_{n}\right\}_{n=1}^{\infty}$ as a set of functions in $A$ (as above) and noting that multiplication is continuous on $A$, we then have

$$
g_{n}=g_{n} \cdot 1=g_{n}\left(p f_{1}+q f_{2}\right)=p\left(g_{n} f_{1}\right)+q\left(g_{n} f_{2}\right),
$$

where this last goes to zero as $n \rightarrow \infty$ since $\left\|g_{n} f_{1}\right\| \rightarrow 0$ and $\left\|g_{n} f_{2}\right\| \rightarrow 0$. But this contradicts the fact that $\left\|g_{n}\right\|=1$ for all $n$; hence it must be that for some $\varepsilon>0$, $\left\|f_{1}(z) g(z)\right\|+\left\|f_{2}(z) g(z)\right\| \geqslant \varepsilon$ whenever $\|g(z)\|=1$, and the sequence $\left\{z^{2 n} \cdot f(z)\right\}_{n=0}^{\infty}$ is basic and equivalent to $\left\{z^{n}\right\}$.

Conversely, suppose $\left|f\left(z_{0}\right)+\right| f\left(-z_{0}\right) \mid=0$ for some $\left|z_{0}\right|=1$. Then for every $n=1,2, \ldots$ let $w_{n}(z)$ and $v_{n}(z)$ be polynomials for which $\left\|f_{1}(z)-w_{n}(z)\right\|<1 / 4 n$, $\left\|f_{2}(z)-v_{n}(z)\right\|<1 / 4 n$, and $w_{n}\left(z_{0}\right)=v_{n}\left(z_{0}\right)=0$, so that $w_{n}(z)=\left(z-z_{0}\right) q_{n}(z)$ and $v_{n}(z)=\left(z-z_{0}\right) t_{n}(z)$ for some polynomials $q_{n}(z)$ and $t_{n}(z)$. If $r$ is any positive integer then

$$
\left(z-z_{0}\right) \cdot \frac{1}{(r+1)}\left(1+\frac{1}{z_{0}} z+\frac{1}{z_{0}^{2}} z^{2}+\cdots+\frac{1}{z_{0}^{r}} z^{r}\right)=\frac{1}{(r+1)}\left(\frac{1}{z_{0}^{r}} z^{r+1}-z_{0}\right)
$$

hence, by the above,

$$
\begin{aligned}
& \left\|w_{n}(z) \cdot \frac{1}{(r+1)}\left(1+\frac{1}{z_{0}} z+\cdots+\frac{1}{z_{0}^{r}} z^{r}\right)\right\| \\
& =\left\|q_{n}(z) \cdot \frac{1}{(r+1)}\left(\frac{1}{z_{0}^{r}} z^{r+1}-z_{0}\right)\right\| \leqslant \frac{2}{(r+1)}\left\|q_{n}(z)\right\| .
\end{aligned}
$$


For every $n$ let $r_{n}$ be an integer for which $2\left(r_{n+1}\right)^{-1}\left\|q_{n}(z)\right\|<1 / 4 n$. Then whenever $r \geqslant r_{n}$,

$$
\left\|w_{n}(z) \cdot \frac{1}{(r+1)}\left(1+\frac{1}{z_{0}} z+\cdots+\frac{1}{z_{0}^{r}} z^{r}\right)\right\|<\frac{1}{4 n} .
$$

Now for every integer $r \geqslant 1$ define the polynomial

$$
p_{r}(z)=\frac{1}{(r+1)}\left(1+\frac{1}{z_{0}} z+\cdots+\frac{1}{z_{0}^{r}} z^{r}\right),
$$

so that $\left\|p_{r}(z)\right\|=1$ for all $r$ and, hence, for which

$$
\left\|\left(f_{1}(z)-w_{n}(z)\right) p_{r}(z)\right\| \leqslant\left\|f_{1}(z)-w_{n}(z)\right\| \cdot\left\|p_{r}(z)\right\|<1 / 4 n
$$

(by definition of $w_{n}(z)$ ). It follows that

$$
\left\|f_{1}(z) p_{r}(z)\right\|<\frac{1}{4 n}+\left\|w_{n}(z) p_{r}(z)\right\|<\frac{1}{4 n}+\frac{1}{4 n}=\frac{1}{2 n} .
$$

An identical argument applied to the polynomials $v_{n}(z)=\left(z-z_{0}\right) t_{n}(z)$ shows that for every $n$ there is an integer $s_{n}$ such that if $r$ is any integer $\geqslant s_{n}$, then one also has $\left\|f_{2}(z) p_{r}(z)\right\|<1 / 2 n$. If for every $n=1,2, \ldots$ we set $i_{n}=\max \left\{r_{n}, s_{n}\right\}$, then it follows that $\left\|f_{1}(z) p_{i_{n}}(z)\right\|<1 / 2 n$ and $\left\|f_{1}(z) p_{i_{n}}(z)\right\|<1 / 2 n$ for all $n$. Hence the sequence $\left\{x_{n}(z)\right\}_{n=1}^{\infty} W(D)$, defined by

$$
x_{n}(z)=\frac{1}{i_{n}+1}\left(1+\frac{1}{z_{0}} z+\cdots+\frac{1}{z_{0}^{i_{n}}} z^{i_{n}}\right),
$$

satisfies $\left\|x_{n}(z)\right\|=1$ and

$$
\left\|T x_{n}\right\|=\left\|f_{1}(z) p_{i_{n}}(z)\right\|+\left\|f_{2}(z) p_{i_{n}}(z)\right\|<1 / 2 n+1 / 2 n=1 / n
$$

for all $n$. That is, $T$ is not bounded below on $W(D)$, and the sequence $\left\{z^{2 n} \cdot f(z)\right\}_{n=0}^{\infty}$ is not equivalent to $\left\{z^{n}\right\}_{n=0}^{\infty}$ in $W(D)$.

The proof of the theorem for $k>2$ is entirely analogous. The condition that $\sum_{i=1}^{k}\left|f\left(\omega^{i}(z)\right)\right| \neq 0$ for all $|z|=1$ can easily be shown to be equivalent to the statement that the $k$ functions

$$
\begin{aligned}
& f_{1}(z)=1+a_{k} z+a_{2 k} z^{2}+\cdots, \\
& f_{2}(z)=a_{1}+a_{k+1} z+a_{2 k+1} z^{2}+\cdots, \\
& f_{k}(z)=a_{k-1}+a_{2 k-1} z+\cdots
\end{aligned}
$$

have no common zero on the circle $|z|=1$. Then using the obvious " $k$-fold" analogue of the argument given above for the case $k=2$, this last condition is shown to be equivalent to the assertion that the operator $T: W(D) \rightarrow W(D)$, defined by $T z^{n}=z^{k n} \cdot f(z)$ for all $n$, is bounded below on $W(D)$, or, what is the same, that $\left\{z^{k n} \cdot f(z)\right\}_{n=0}^{\infty}$ is a basic sequence in $W(D)$ equivalent to $\left\{z^{n}\right\}_{n=0}^{\infty}$.

Finally, the case $k=1$ can be treated even more simply. Here the condition $f(z) \neq 0$ for all $|z|=1$ is equivalent (by the Wiener-Levy Theorem [3, p. 196]) to the statement that $f^{-1}(z) \in A$, and this latter is easily shown to be equivalent to the fact that $T: W(D) \rightarrow W(D)$, defined by $T\left(z^{n}\right)=z^{n} f(z)$, is bounded below on $l^{1}$. 
3. Remarks. The theorem given in $\$ 2$ provides a complete function-theoretic description of the shift basic sequences in $W(D)$ which are equivalent to $\left\{z^{n}\right\}_{n=0}^{\infty}$. In view of this result it is natural to conjecture that arbitrary shift basic sequences in $W(D)$ are also characterized by properties of the zeros of their generating functions on the circle $|z|=1$. Further evidence to support this conjecture is provided by our earlier paper [2], where it is shown that in the case where $f(z)$ is a polynomial and $k=1$ the 1-shifted sequence $\left\{z^{n} \cdot f(z)\right\}_{n=0}^{\infty}$ is a basic sequence in $W(D)$ if and only if the zeros of $f(z)$ on the circle $|z|=1$ are all simple. As the nature of this result suggests, the treatment of the general case where $f(z)$ is arbitrary, even when $k=1$, is not immediate. Using the observations about $k$-shifted sequences made in $\S 2$, together with the techniques of [2], one can give a characterization of the basicity of $k$-shifted sequences $\left\{z^{k n} \cdot f(z)\right\}_{n=0}^{\infty}$ when $f(z)$ is a polynomial. This and other related results concerning shift sequences will be given in a forthcoming paper.

\section{REFERENCES}

1. H. Helson and G. Szegö, A problem in prediction theory, Ann. Mat. Pura Appl. 51 (1960), 107-138.

2. J. Holub, On bases and the shift operator, Studia Math. 71 (1981), 191-202.

3. M. Naĭmark, Normed rings, Noordhoff, Groningen, 1964.

4. I. Singer, Bases in Banach spaces. I, Springer-Verlag, Berlin, 1970.

Department of Mathematics, Virginia Polytechnic Institute and State University, BlacksBURG, VIRGINIA 24061 